\title{
Mixed convection flow over a vertical wedge embedded in a highly porous medium
}

\author{
M. Kumari, H. S. Takhar, G. Nath
}

\begin{abstract}
The steady mixed convection flow over a vertical $\mathrm{Nu}, \mathrm{Pr}$ wedge with a magnetic field embedded in a porous medium has been investigated. The effects of the permeability of the medium, surface mass transfer and viscous dissipation on the flow and temperature fields have been included in the analysis. The coupled nonlinear partial differential equations governing the flow field have been solved numerically using the Keller box method. The skin friction and heat transfer are found to increase with the parameters characterizing the permeability of the medium, buoyancy force, magnetic field and pressure gradient.

However the effect of the permeability and magnetic field on the heat transfer is very small. The heat transfer increases with the Prandtl number, but the skin friction decreases. The buoyancy force which assists the forced convection flow causes an overshoot in the velocity profiles. Both the skin friction and heat transfer increase with suction and the effect of injection is just the reverse.

\section{List of symbols}

$B \quad$ magnetic field

$c_{\mathrm{p}} \quad$ specific heat at a constant pressure

$C_{\mathrm{f}} \quad$ skin friction coefficient

E $\quad$ Eckert number

$f \quad$ dimensionless stream function

$g \quad$ acceleration due to gravity

$G$ dimensionless temperature

$\mathrm{Gr}_{L} \quad$ Grashof number

$\mathrm{Ha} \quad$ Hartmann number

$K, K_{2} \quad$ dimensionless permeability parameters

$K_{1} \quad$ dimensional permeability parameter

$L, M \quad$ characteristic length and magnetic parameter, respectively

$m$ index in the power-law variation of the velocity at the edge of the boundary layer

$N_{1}, N_{2}, N_{3}$ defined in Eq. (9)

$N_{4}, N_{5}, N_{6}$ defined in Eq. (13)
\end{abstract}

Received on 21 May 1999

M. Kumari, H. S. Takhar, G. Nath

Department of Mathematics

Indian Institute of Science

Bangalore - 560 012, India

H. S. Takhar $(\bowtie)$

Department of Engineering

Manchester Metropolitan University

Oxford Road, Manchester, M1 5GD, UK
Nusselt number and Prandtl number, respectively

$\mathrm{Re}_{L}, \mathrm{Re}_{x} \quad$ Reynolds numbers defined with respect

to $L$ and $x$, respectively

constant

$s$

$T$

$u, v$

$x, y$

dimensional temperature

velocity components along $x$ and $y$ directions, respectively

distances along and perpendicular to the surface

Greek symbols

$\alpha \quad$ ratio of Grashof number and Reynolds number squared

$\beta \quad$ pressure gradient parameter

$\beta_{1} \quad$ coefficient of thermal expansion

$\xi, \eta \quad$ transformed coordinates

$v \quad$ kinematic viscosity

$\rho, \sigma \quad$ density and electrical conductivity, respectively

$\psi \quad$ dimensional stream function

Superscript

, derivative with respect to $\eta$

Subscripts

e, $w \quad$ conditions at the edge of the boundary

layer and on the wall, respectively

$x, y, \xi \quad$ derivatives with respect to $x, y$ and $\xi$, respectively

$\infty \quad$ free stream conditions

\section{1}

\section{Introduction}

Convective heat transfer from surfaces embedded in porous media has been the topic of several studies in recent years. The interest for such studies is motivated by a wide range of thermal engineering applications, such as geothermal systems, oil extraction, ground water pollution, thermal insulation, solid matrix heat exchangers and the storage of nuclear wastes. Comprehensive reviews of the above studies were published by Chang $(1978,1985)$. The transport of momentum and thermal energy in fluid saturated porous media with low porosities, such as rocks, soil, sand etc., is described by Darcy's model. For lowporosity media the entry region as well as the boundary layer effect are generally small, hence the convective and viscous terms in the momentum equations can be neglected (Vafai and Tien, 1981). However, for highly porous media such as foam, metals and fibrous media, this is not 
the case. Hence the convective and viscous terms have to be retained in the momentum equations. The steady free convection flow over a vertical flat plate in the fluid saturated porous media with low porosities using Darcy's model has been studied by Cheng and Minkowycz (1977), Bejan and Khair (1985), Ingham and Pop (1987), Merkin and Pop (1987), Ram (1988), and Chen and Chen (1988). Also Bejan (1987) has presented an excellent review of the free convection flow in saturated porous media. The steady free convection flow over a vertical plate in highly porous media taking into account the convective and viscous terms in the momentum equation has been investigated by Raptis and Kofousias (1982) and Raptis (1986). However, there have been only a few studies dealing with the mixed convection flow over a vertical plate in porous media. The mixed convection over inclined surfaces in a saturated porous medium with low porosity using Darcy's model has been studied by Cheng (1977). Takhar and Perdikis (1986) and Raptis and Perdikis (1988) have considered the mixed convection flow over a vertical plate in a highly porous medium.

The present analysis deals with the mixed convection flow over a vertical wedge with a magnetic field embedded in a highly porous medium. The effects of mass transfer, viscous dissipation and Joule heating have been included in the analysis. The partial differential equations have been solved numerically using the Keller box method (Cebeci and Bradshaw, 1984). The particular cases of the present results have been compared with those available in the literature. The reason for studying the effect of the magnetic field on the flow through porous media is that the fluids are electrically conducting in geothermic regions and hence these can be significantly influenced by the magnetic field (Cheng, 1978).

\section{2}

\section{Governing equations}

Let us consider the steady mixed convection flow of an electrically conducting fluid with an applied magnetic field $B$ (fixed relative to the fluid) in the $y$ direction over the surface of a vertical wedge with included angle $\pi \beta$ immersed in porous medium (Fig. 1). The effects of the permeability of the medium, magnetic field, buoyancy parameter, mass transfer and viscous and ohmic dissipation on the flow and temperature fields have been considered. The magnetic Reynolds number is assumed to be small (for most problems, this assumption is true) which implies that the induced magnetic field can be neglected in comparison to the applied magnetic field. However, the Hall effect has been neglected. The wall temperature and the free stream temperature are taken as constants. In a body fixed coordinate system with $x$ measured along the surface of the wedge from the apex, the velocity at the edge of the boundary layer is given by $u_{\mathrm{e}}=u_{\infty}(x / L)^{m}$ where the constant $m$ is related to the included angle of the wedge $\pi \beta$, by $m=\beta /(2-\beta)$. The porous medium is considered to be isotropic and homogeneous and it causes the flow resistance which is taken to be proportional to the velocity. Here we assume that all the flow properties are constant except the density which gives rise to the buoyancy force. Under the foregoing assumptions, the boundary layer

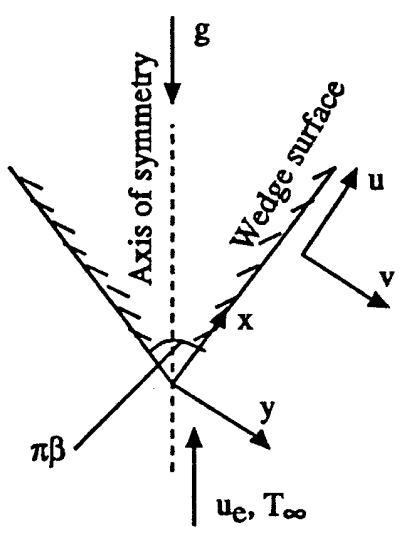

Fig. 1. Physical model and coordinate system

equations governing the nonsimilar flow over a wedge embedded in a highly porous medium can be expressed as (Bejan, 1987; Cheng, 1978; Vafai and Tein, 1981);

$u_{x}+v_{y}=0$,

$$
\begin{aligned}
u u_{x}+v u_{y}= & u_{\mathrm{e}}\left(u_{\mathrm{e}}\right)_{x}+v u_{y y}+g \beta_{1}\left(T-T_{\infty}\right) \cos (\pi \beta / 2) \\
& -\left(\sigma B^{2} / \rho\right)\left(u-u_{\mathrm{e}}\right) \\
& -\left(g / K_{1}\right)\left(u-u_{\mathrm{e}}\right) \cos (\pi \beta / 2) \\
u T_{x}+v T_{y}= & (v / \operatorname{Pr}) T_{y y}+\left(v / c_{\mathrm{p}}\right) u_{y}^{2}+\left(\sigma B^{2} / \rho c_{\mathrm{p}}\right) u^{2}
\end{aligned}
$$

Equations (1)-(3) are subjected to the following boundary conditions:

$$
\begin{aligned}
& u=0, v=v_{\mathrm{w}}, T=T_{\mathrm{w}} \quad \text { at } y=0, x \geq 0, \\
& u \rightarrow u_{\mathrm{e}}, T \rightarrow T_{\infty} \quad \text { as } y \rightarrow \infty, x \geq 0 \\
& u=u_{\mathrm{e}}, T=T_{\infty} \quad \text { at } x<0, y \geq 0
\end{aligned}
$$

Equations (1)-(4) are transformed from $(x, y)$ coordinates to the pseudo-similarity variable $\eta$ and $x$-dependent dimensionless variable $\bar{x}$ by using the following transformations;

$$
\begin{aligned}
& \eta=y\left[(m+1 / 2)\left(u_{\mathrm{e}} / v x\right)\right]^{1 / 2}, \\
& \psi=\left[(2 / m+1) v x u_{\mathrm{e}}\right]^{1 / 2} f(\bar{x}, \eta) \\
& u=\psi_{y}, \quad v=-\psi_{x}, \quad u_{\mathrm{e}}=u_{\infty} \bar{x}^{m}, \bar{x}=x / L, \\
& u=u_{\mathrm{e}} f^{\prime}(\bar{x}, \eta) \\
& -v=\left[(2 / m+1)\left(v u_{\mathrm{e}} / x\right)\right]^{1 / 2} \times[f / 2 \\
& \left.+(1 / 2)\left(x / u_{\mathrm{e}}\right)\left(\mathrm{d} u_{\mathrm{e}} / \mathrm{d} x\right) f+x\left(f_{x}+f^{\prime} \eta_{x}\right)\right] \\
& G=\left(T-T_{\infty}\right) /\left(T_{\mathrm{w}}-T_{\infty}\right) .
\end{aligned}
$$

We find that Eq. (1) is satisfied identically and Eqs. (2) and (3) reduce to

$$
\begin{aligned}
& f^{\prime \prime \prime}+f f^{\prime \prime}+(2 m / m+1)\left(1-f^{\prime 2}\right) \\
&+(2 / m+1) \alpha N_{1} \cos (\pi \beta / 2) G \\
&+(2 / m+1) N_{2}[M+K \cos (\pi \beta / 2)]\left(1-f^{\prime}\right) \\
&=(2 / m+1) \bar{x}\left(f_{\bar{x}}^{\prime} f^{\prime}-f_{\bar{x}} f^{\prime \prime}\right), \\
& \operatorname{Pr}^{-1} G^{\prime \prime}+f G^{\prime}+E\left[N_{3} f^{\prime \prime 2}+(2 / m+1) N_{2} M f^{\prime 2}\right] \\
& \quad=(2 / m+1) \bar{x}\left(G_{\bar{x}} f^{\prime}-f_{\bar{x}} G^{\prime}\right) .
\end{aligned}
$$


The boundary conditions (4) can be re-written as

$f=f_{\mathrm{w}}, f^{\prime}=0, G=1 \quad$ at $\eta=0, \bar{x} \geq 0$,

$f^{\prime} \rightarrow 1, G \rightarrow 0$ as $\eta \rightarrow \infty, \bar{x} \geq 0$,

$f^{\prime}=1, G=0$ for $\bar{x}<0, \eta \geq 0$,

where

$$
\begin{aligned}
& m=\left(\bar{x} / u_{\mathrm{e}}\right)\left(\mathrm{d} u_{\mathrm{e}} / \mathrm{d} \bar{x}\right), \quad N_{1}=\bar{x}\left(u_{\infty} / u_{\mathrm{e}}\right)^{2}, \\
& N_{2}=\bar{x}\left(u_{\infty} / u_{\mathrm{e}}\right), \quad N_{3}=\left(u_{\mathrm{e}} / u_{\infty}\right)^{2}, \\
& \alpha=\mathrm{Gr}_{L} / \operatorname{Re}_{L}^{2}, \quad M=\mathrm{Ha}^{2} / \operatorname{Re}_{L}, \\
& E=u_{\infty}^{2} /\left[c_{\mathrm{p}}\left(T_{\mathrm{w}}-T_{\infty}\right)\right], \\
& K=K_{2} / \operatorname{Re}_{L}, \quad K_{2}=g L^{2} /\left(v K_{1}\right), \quad \operatorname{Re}_{L}=u_{\infty} L / v, \\
& \mathrm{Gr}_{L}=g \beta_{1}\left(T_{\mathrm{w}}-T_{\infty}\right) L^{3} / v^{2}, \quad \mathrm{Ha}^{2}=\sigma B^{2} L^{2} / \mu, \\
& \operatorname{Re}_{x}=u_{\mathrm{e}} x / v, \\
& \left.-v_{\mathrm{w}}[(m+1) / 2\}\left(\bar{x} L / v u_{\mathrm{e}}\right)\right]^{1 / 2} \\
& \quad=\left[f_{\mathrm{w}} / 2+(1 / 2)\left(\bar{x} / u_{\mathrm{e}}\right)\left(\mathrm{d} u_{\mathrm{e}} / \mathrm{d} \bar{x}\right) f_{\mathrm{w}}+\bar{x}\left(\mathrm{~d} f_{\mathrm{w}} / \mathrm{d} \bar{x}\right)\right] .
\end{aligned}
$$

The local skin friction and heat transfer (Nusselt number) coefficients can be expressed as

$$
\begin{aligned}
& C_{\mathrm{f}}=2 \mu\left(u_{y}\right)_{\mathrm{w}} / \rho u_{\mathrm{e}}^{2}=[2(m+1)]^{1 / 2}\left(\mathrm{Re}_{x}\right)^{-1 / 2}\left(f^{\prime \prime}\right)_{\mathrm{w}}, \\
& \mathrm{Nu}=-x\left(T_{y}\right)_{\mathrm{w}} /\left(T_{\mathrm{w}}-T_{\infty}\right) \\
& \quad=-[(m+1) / 2]^{1 / 2}\left(\operatorname{Re}_{x}\right)^{1 / 2}\left(G^{\prime}\right)_{\mathrm{w}} .
\end{aligned}
$$

If we put $\xi=\bar{x}^{(1-m) / 2}$, then Eqs. (6) and (7) reduce to

$$
\begin{aligned}
& f^{\prime \prime \prime}+f f^{\prime \prime}+(2 m / m+1)\left(1-f^{\prime 2}\right) \\
&+(2 / m+1) \alpha N_{4} G \cos (\pi \beta / 2) \\
&+(2 / m+1)[M+K \cos (\pi \beta / 2)] N_{5}\left(1-f^{\prime}\right) \\
&= {[(1-m) /(1+m)] \xi\left(f_{\xi}^{\prime} f^{\prime}-f_{\xi} f^{\prime \prime}\right), } \\
& \operatorname{Pr}^{-1} G^{\prime \prime}+f G^{\prime}+E N_{6}\left[f^{\prime \prime 2}+(2 / m+1) N_{5} M f^{\prime 2}\right] \\
&=[(1-m) /(1+m)] \xi\left(G_{\xi} f^{\prime}-f_{\xi} G^{\prime}\right),
\end{aligned}
$$

where

$N_{4}=\xi^{2(1-2 m) /(1-m)}, \quad N_{5}=\xi^{2}, \quad N_{6}=\xi^{4 m /(1-m)}$.

The boundary conditions are:

$f=f_{\mathrm{w}}, f^{\prime}=0, G=1$ at $\eta=0, \xi \geq 0$,

$f^{\prime} \rightarrow 1, G \rightarrow 0$ as $\eta \rightarrow \infty, \xi \geq 0$,

$f^{\prime}=1, G=0$ for $\xi<0, \eta \geq 0$,

where $f_{\mathrm{w}}$ is given by

$s=[(1+m) / 2] f_{\mathrm{w}}+[(1-m) / 2] \xi\left(\partial f_{\mathrm{w}} / \partial \xi\right)$,

and the mass transfer parameter $s$ is expressed as

$s=-\left[(1+m) 2^{-1}\left(\operatorname{Re}_{L}\right)\right]^{1 / 2}\left(v_{\mathrm{w}} / u_{\infty}\right)(x / L)^{(1-m) / 2}$.

If $\left(v_{\mathrm{w}} / u_{\infty}\right)$ varies as $(x / L)^{(m-1) / 2}$, then the mass transfer parameter $s$ is a constant because $u_{\infty}, \operatorname{Re}_{L}, m$ and $L$ are all constants.

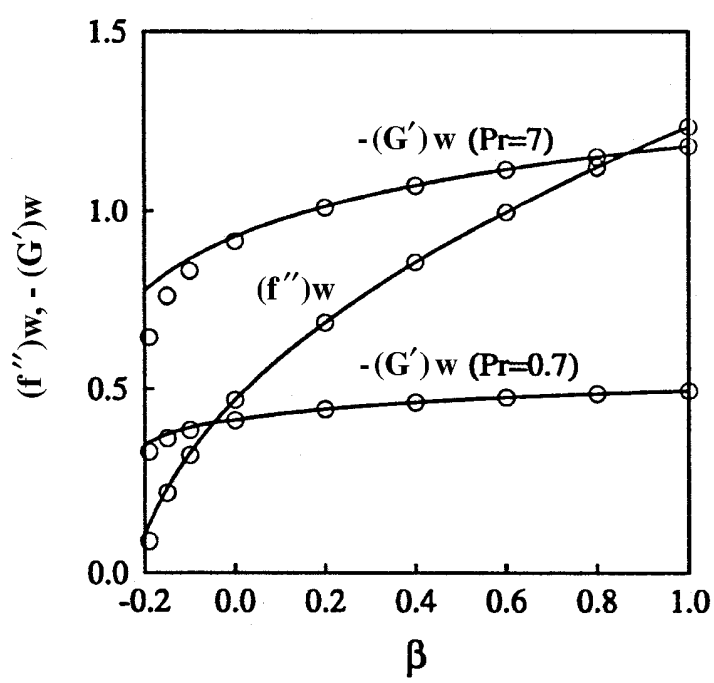

Fig. 2. Skin-friction parameter $\left(f^{\prime \prime}\right)_{\mathrm{w}}$ and heat-transfer parameter $-\left(G^{\prime}\right)_{\mathrm{w}}$ for $\xi=M=\alpha=K=s=E=0$. _ : Present results; o: Evans (1968)

Table 1. Comparison of heat transfer results $\left(-\left(G^{\prime}\right)_{\mathrm{w}}\right)$ with those of Raptis and Perdikis (1988) for the flat plate when $\xi=M=\beta=E=0, \operatorname{Pr}=0.72, \alpha=0.2$

\begin{tabular}{lrll}
\hline$K$ & $f_{\mathrm{w}}$ & $-\left(G^{\prime}\right)_{\mathrm{w}}$ & \\
\cline { 3 - 4 } & & $\begin{array}{l}\text { Present } \\
\text { results }\end{array}$ & $\begin{array}{l}\text { Raptis and } \\
\text { Perdikis (1988) }\end{array}$ \\
\hline 1 & -0.5 & 0.2672 & 0.267 \\
1 & 0.5 & 0.4955 & 0.492 \\
2 & -0.5 & 0.2529 & 0.252 \\
2 & 0.5 & 0.4845 & 0.481 \\
\hline
\end{tabular}

Table 2. Comparison of skin friction $\left(\left(f^{\prime \prime}\right)_{\mathrm{w}}\right)$ and heat transfer $\left(-\left(G^{\prime}\right)_{\mathrm{w}}\right)$ results with those of Watanabe (1990) for the wedge when $\alpha=M=K=s=E=0, \operatorname{Pr}=0.73$

\begin{tabular}{llllll}
\hline$m$ & $\left(f^{\prime \prime}\right)_{\mathrm{w}}$ & & & $-\left(G^{\prime}\right)_{\mathrm{w}}$ & \\
& $\begin{array}{l}\text { Present } \\
\text { results }\end{array}$ & $\begin{array}{l}\text { Watanabe } \\
(1990)\end{array}$ & & $\begin{array}{l}\text { Present } \\
\text { results }\end{array}$ & $\begin{array}{l}\text { Watanabe } \\
(1990)\end{array}$ \\
\hline 0 & 0.46975 & 0.46960 & & 0.42079 & 0.42015 \\
0.0141 & 0.50472 & 0.50461 & & 0.42635 & 0.42578 \\
0.0435 & 0.56904 & 0.56898 & & 0.43597 & 0.43548 \\
0.0909 & 0.65501 & 0.65498 & & 0.44770 & 0.44730 \\
0.1429 & 0.73202 & 0.73200 & & 0.45728 & 0.45693 \\
0.2000 & 0.80214 & 0.80213 & & 0.46534 & 0.46503 \\
0.3333 & 0.92766 & 0.92765 & & 0.47840 & 0.47814 \\
\hline
\end{tabular}

The expressions for skin friction and heat transfer coefficients become

$C_{\mathrm{f}}\left(\operatorname{Re}_{x}\right)^{1 / 2}=[2(1+m)]^{1 / 2}\left(f^{\prime \prime}\right)_{\mathrm{w}}$,

$\mathrm{Nu}\left(\operatorname{Re}_{x}\right)^{-1 / 2}=-[(1+m) / 2]^{1 / 2}\left(G^{\prime}\right)_{\mathrm{w}}$.

It may be noted from Eq. (15) that the surface mass transfer $f_{\mathrm{w}}(=s)$ is a constant. It has been assumed that the injected fluid possesses the same physical properties as the 


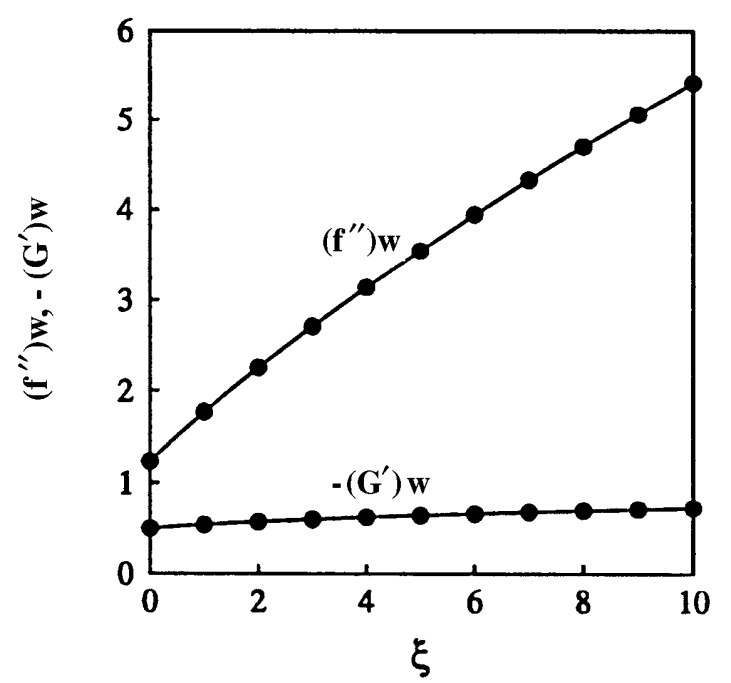

Fig. 3. Skin-friction parameter $\left(f^{\prime \prime}\right)_{\mathrm{w}}$ and heat-transfer parameter $-\left(G^{\prime}\right)_{\mathrm{w}}$ for $\beta=1, M=s=\alpha=K=E=0$ and $\operatorname{Pr}=0.7$.

Present results; •: Ramachandran et al. (1988)
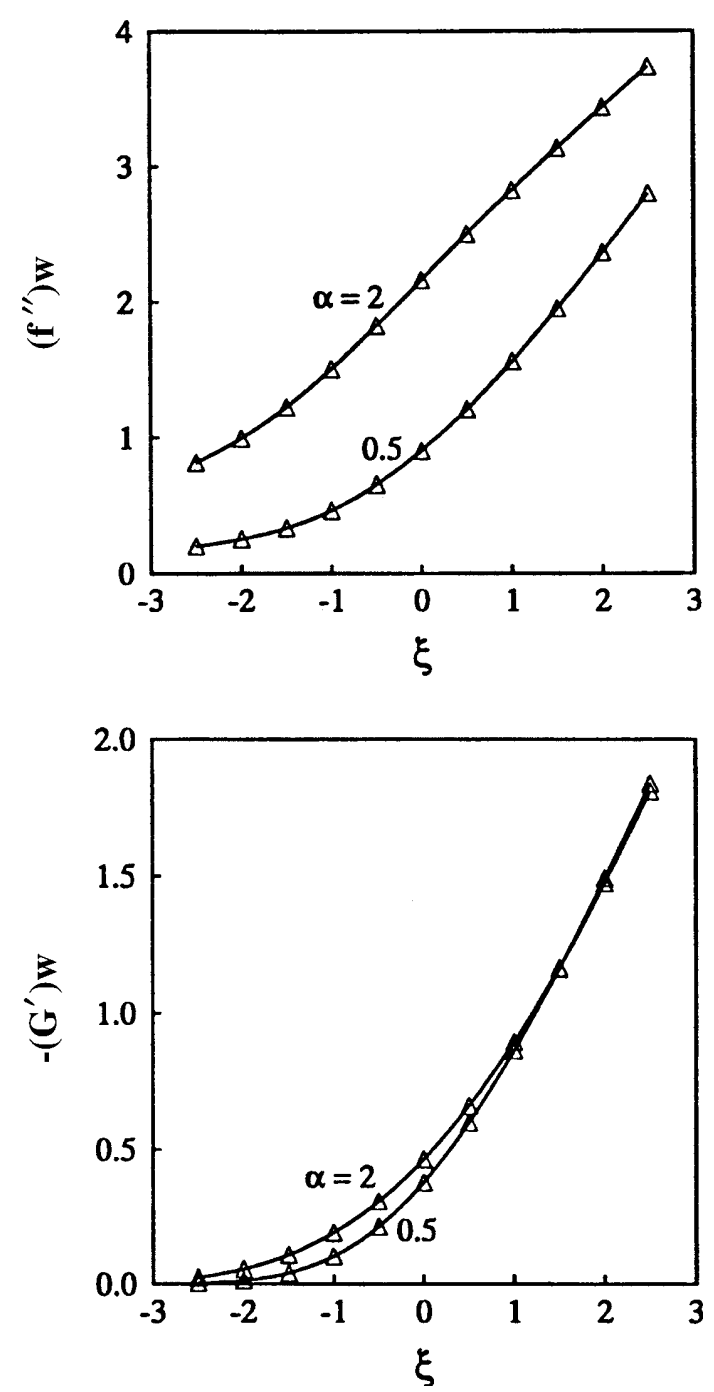

Fig. 4. Skin-friction parameter $\left(f^{\prime \prime}\right)_{\mathrm{w}}$ and heat-transfer parameter $-\left(G^{\prime}\right)_{\mathrm{w}}$ for $M=K=E=m=0$. : Present results; $\triangle$ : Watanabe (1991) boundary layer fluid and has a static temperature equal to the wall temperature.

It may be remarked that Eqs. (11) and (12) with boundary conditions (14) govern the steady nonsimilar mixed convection flow over a vertical wedge. For $\xi=M=K=\alpha=0$, Eq. (11) reduces to the well known Falkner-Skan equation which represents the similarity equation governing the forced convection flow over a wedge and Eq. (12) represents the temperature field for constant wall temperature case. Also for

$\beta=m=\xi=M=E=0, N_{4}=N_{5}=1$, Eqs. (11) and (12) reduce to those of Raptis and Perdikis (1988), who considered the mixed convection flow over a vertical plate, if we replace $f$ by $(2)^{-1 / 2} f, \eta$ by $(2)^{-1 / 2} \eta, K$ by $2 / K$, $\alpha$ by $2 \mathrm{Gr}_{x} / \operatorname{Re}_{x}^{2}$ and $G$ by $\theta$. Furthermore, for $\xi=M=K=E=0, \beta=m=1$, Eqs. (11) and (12) reduce to those of Ramachandran et al. (1988) who considered the mixed convection flow in the stagnation region of a vertical surface with constant wall temperature $(n=0)$. However they have denoted the buoyancy parameter by $\xi$ instead of $\alpha_{1}$. For $\alpha=M=K=E=0$, Eqs. (6) and (7) or
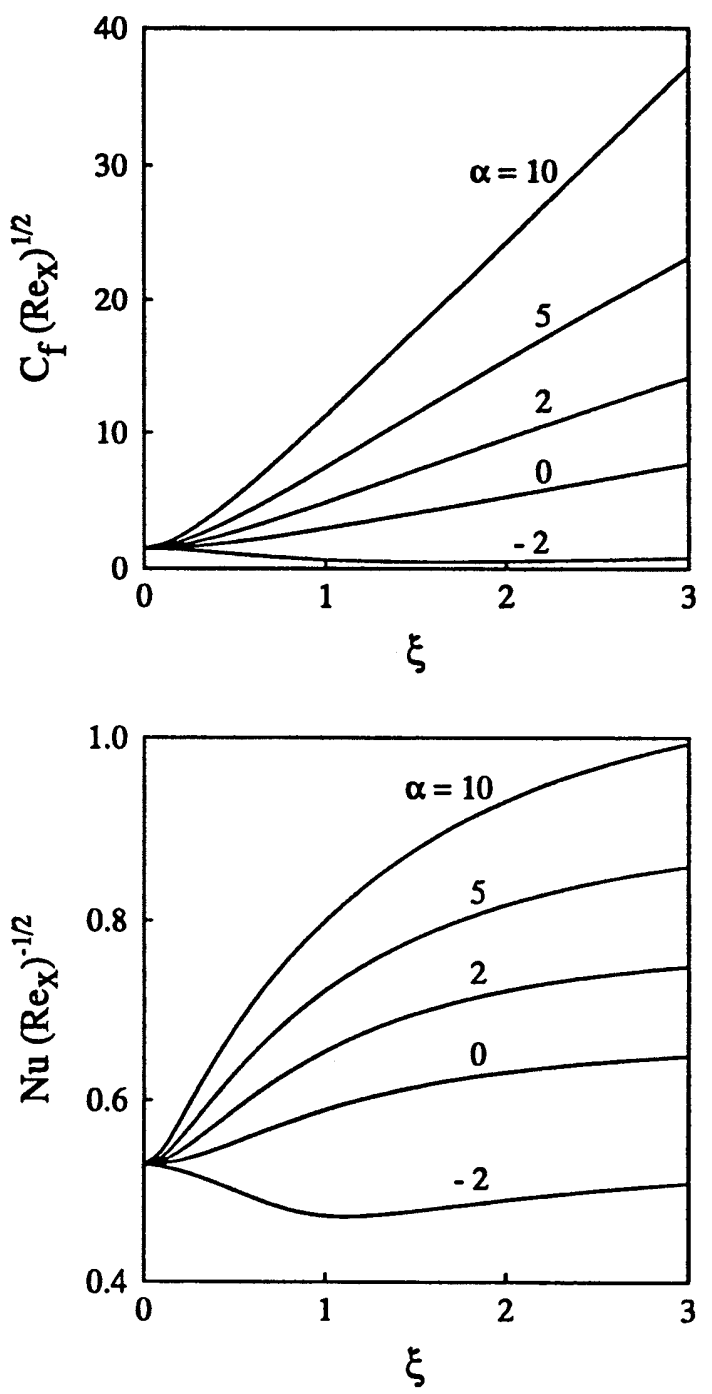

Fig. 5. Skin-friction coefficient $C_{\mathrm{f}}\left(\operatorname{Re}_{x}\right)^{1 / 2}$ and heat-transfer coefficient $\mathrm{Nu}\left(\mathrm{Re}_{x}\right)^{-1 / 2}$ for $K=s=0.5, m=0.0909, M=1$, $E=0$ and $\operatorname{Pr}=0.72$ 
(11) and (12) reduce to those of Watanabe (1990) who considered the forced convection flow over a wedge and for $M=K=E=m=\beta=0$, Eqs. (6) and (7) or (11) and (12) reduce to those of Watanabe (1991) who considered the mixed convection flow on a vertical flat plate if we replace $f$ by $2^{-1 / 2} f, \eta$ by $2^{-1 / 2} \eta$.

\section{3}

\section{Results and discussion}

The partial differential equations (11) and (12) under boundary conditions (14) have been solved using the Keller box method. Since the method is described by Cebeci and Bradshaw (1984), it is not presented here. We have studied the effect of step size $\Delta \eta$ and $\Delta \xi$ and the edge of the boundary layer $\eta_{\infty}$ on the solution in order to optimize them. The results presented here are independent of step size and $\eta_{\infty}$ at least up to the 5th decimal place.

The validity of the present solutions is examined by comparing the results for the skin friction and heat transfer $\left(\left(f^{\prime \prime}\right)_{\mathrm{w}},-\left(G^{\prime}\right)_{\mathrm{w}}\right)$ with those of well known
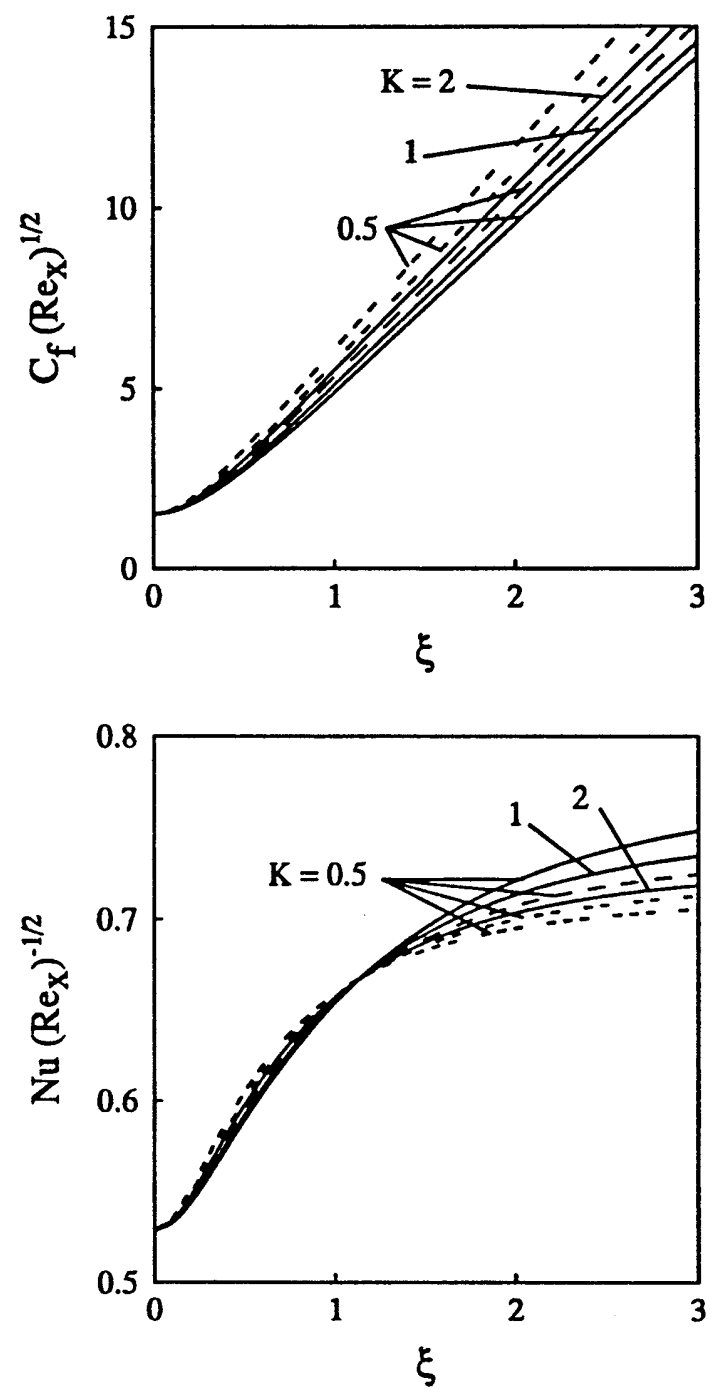

Fig. 6. Skin-friction coefficient $C_{\mathrm{f}}\left(\operatorname{Re}_{x}\right)^{1 / 2}$ and heat-transfer coefficient $\mathrm{Nu}\left(\mathrm{Re}_{x}\right)^{-1 / 2}$ for $s=0.5, m=0.0909, \alpha=2, E=0$ and $\operatorname{Pr}=0.72$. $: M=1 ;---: M=2 ;----: M=3$;
Falkner-Skan equation when $\xi=M=K=\alpha=E=0$ (Evans, 1968). The results are found to be in excellent agreement for accelerated flows $(\beta \geq 0)$ and for retarded flows $(\beta<0),\left(f^{\prime \prime}\right)_{\mathrm{w}}$ and $-\left(G^{\prime}\right)_{\mathrm{w}}$ differ by approximately $1-53 \%$ and $1-8 \%$ respectively as $\beta$ varies from -0.1 to -0.19 (Fig. 2). Also the results were compared with those of Raptis and Perdikis (1988) when

$\beta=m=\xi=M=E=0, N_{4}=N_{5}=1$, and with those of Ramachandran et al. (1988) when $\xi=M=K=E=0$, $\beta=m=1$. For $\alpha=M=K=E=0$, the results have been compared with Watanabe (1990) and for $M=K=E=m=\beta=0$ a comparison has been made with the results of Watanabe (1991). The results are found to be in excellent agreement. The comparison is shown in Tables 1 and 2 and Figs. 3 and 4. The computations have been carried out for various values of the parameters $m, \alpha$, $M, K, s, \operatorname{Pr}$ and $E$. However the results are presented here only for some representative values of these parameters.

Figure 5 shows the effect of the buoyancy parameter $\alpha$ on the skin friction and heat transfer coefficients
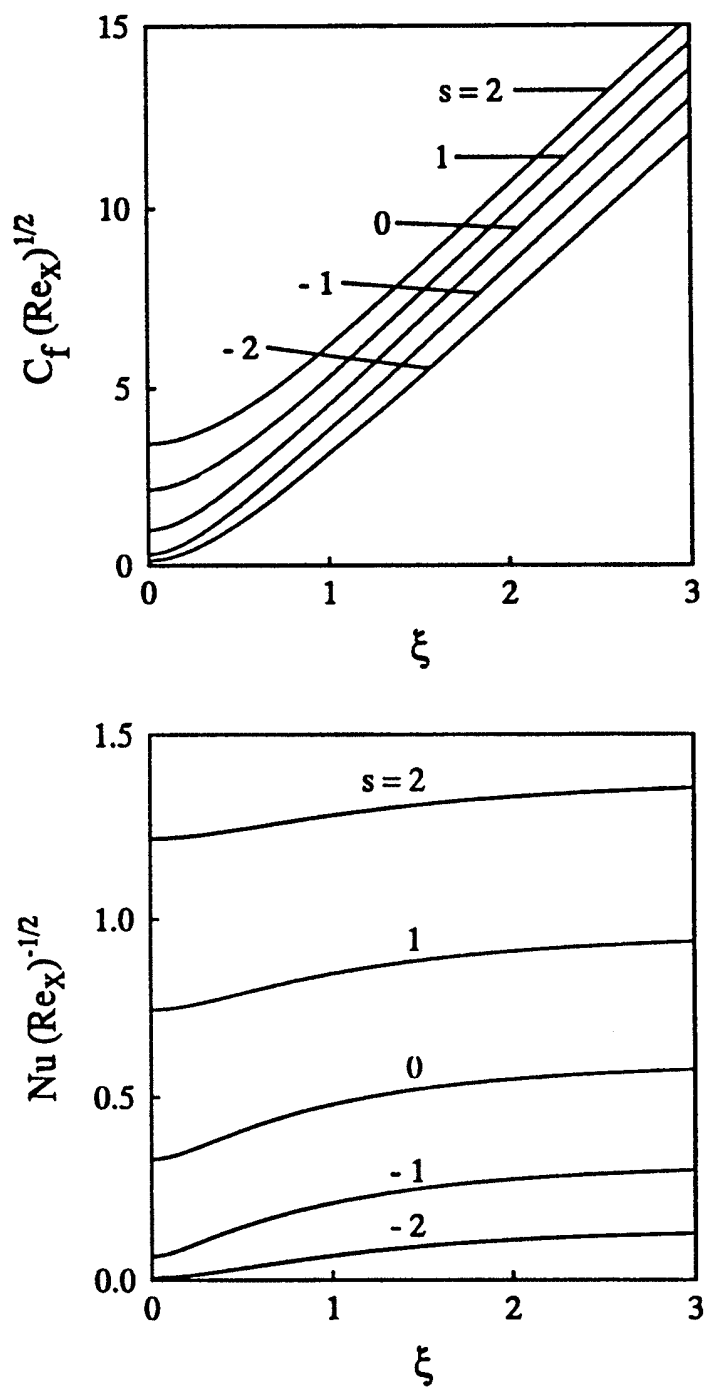

Fig. 7. Skin-friction coefficient $C_{\mathrm{f}}\left(\mathrm{Re}_{x}\right)^{1 / 2}$ and heat-transfer coefficient $\mathrm{Nu}\left(\mathrm{Re}_{x}\right)^{-1 / 2}$ for $K=0.5, m=0.0909, M=1, \alpha=2$, $E=0$ and $\operatorname{Pr}=0.72$ 
$\left(C_{\mathrm{f}}\left(\mathrm{Re}_{x}\right)^{1 / 2}, \mathrm{Nu}\left(\mathrm{Re}_{x}\right)^{-1 / 2}\right)$. When $\alpha>0$ (aiding flow), both the skin friction and heat transfer coefficients are enhanced as $\alpha$ increases. However, for the opposing flow $(\alpha<0)$, the trend is just the opposite. The reason for such a behaviour is that the buoyancy parameter $\alpha(\alpha>0)$ acts as a favourable pressure gradient. Consequently, the fluid within the boundary layer is accelerated and both the momentum and the thermal boundary layer thicknesses are reduced. The net result is that both $C_{\mathrm{f}}\left(\mathrm{Re}_{x}\right)^{1 / 2}$ and $\mathrm{Nu}\left(\mathrm{Re}_{x}\right)^{-1 / 2}$ are increased. It is observed that the effect of the buoyancy parameter $\alpha$ is more pronounced on the skin-friction coefficient $\left(C_{\mathrm{f}}\left(\mathrm{Re}_{x}\right)^{1 / 2}\right)$ than on the heat-transfer coefficient $\left(\mathrm{Nu}\left(\mathrm{Re}_{x}\right)^{-1 / 2}\right)$. The skin-friction and heat-transfer coefficients increase with the streamwise distance $\xi$ for aiding flow $(\alpha>0)$. For opposing flow $(\alpha<0)$, the skin-friction coefficient decreases with $\xi$ whereas the heat-transfer coefficient first decreases and then increases with the streamwise distance $\xi$.

The effects of the magnetic parameter $M$ and the permeability parameter $K$ on the skin-friction and
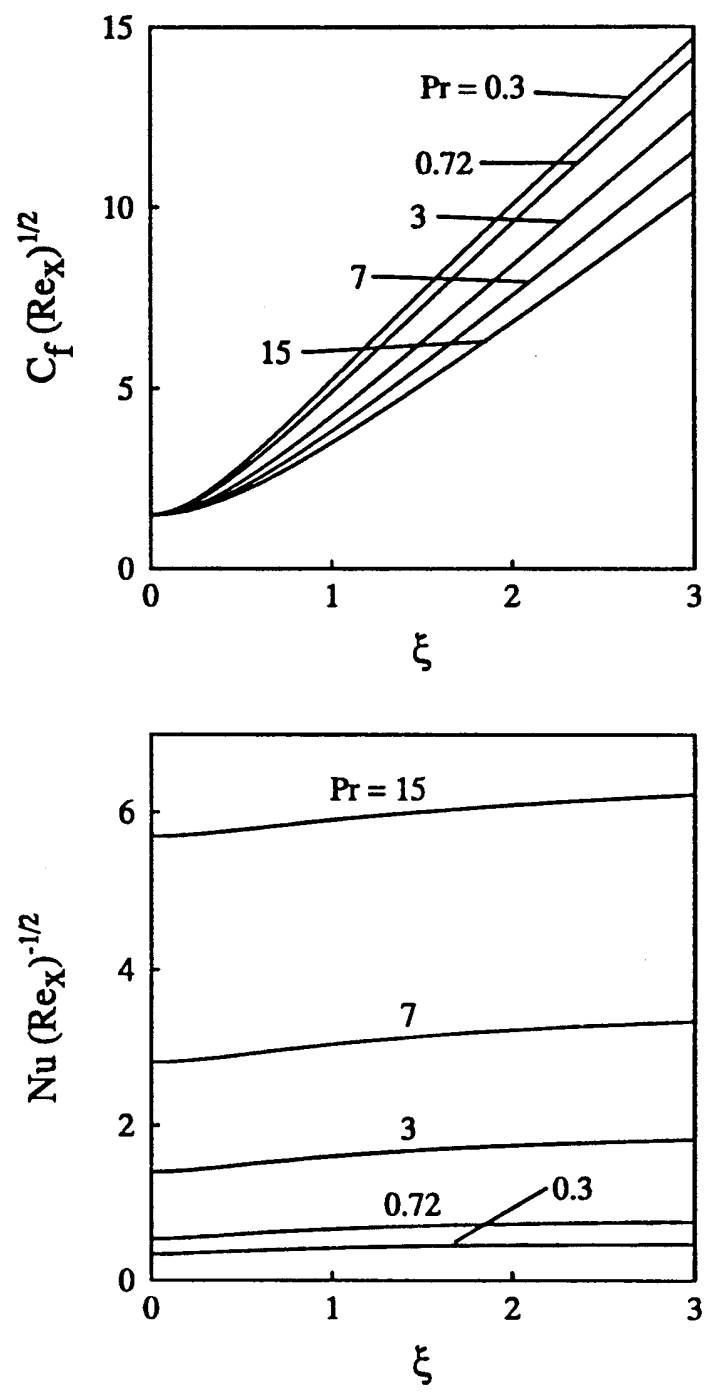

Fig. 8. Skin-friction coefficient $C_{\mathrm{f}}\left(\operatorname{Re}_{x}\right)^{1 / 2}$ and heat-transfer coefficient $\mathrm{Nu}\left(\mathrm{Re}_{x}\right)^{-1 / 2}$ for $K=s=0.5, m=0.0909, M=1$, $\alpha=2$ and $E=0$ heat-transfer coefficients $\left(C_{\mathrm{f}}\left(\mathrm{Re}_{x}\right)^{1 / 2}, \mathrm{Nu}\left(\mathrm{Re}_{x}\right)^{-1 / 2}\right)$ are displayed in Fig. 6. It is observed that the skin-friction coefficient $C_{\mathrm{f}}\left(\mathrm{Re}_{x}\right)^{1 / 2}$ increases as $M$ or $K$ increases. However, the heat-transfer coefficient $\left(\mathrm{Nu}\left(\mathrm{Re}_{x}\right)^{-1 / 2}\right)$ is found to be weakly dependent on $M$ and $K$. Also they increase with the streamwise distance $\xi$. The reason for the weak dependence of the heat-transfer coefficient $\mathrm{Nu}\left(\mathrm{Re}_{x}\right)^{-1 / 2}$ on $M$ or $K$ is that these parameters do not occur explicitly in the equation governing $G$ unlike the momentum equation.

The effect of the mass transfer $f_{\mathrm{w}}(s)$ on the skin-friction and heat-transfer coefficients $\left(C_{\mathrm{f}}\left(\mathrm{Re}_{x}\right)^{1 / 2}, \mathrm{Nu}\left(\mathrm{Re}_{x}\right)^{-1 / 2}\right)$ is presented in Fig. 7. The skin-friction coefficient $\left(C_{\mathrm{f}}\left(\mathrm{Re}_{x}\right)^{1 / 2}\right)$ and heat-transfer coefficient $\left(\mathrm{Nu}\left(\mathrm{Re}_{x}\right)^{-1 / 2}\right)$ are enhanced by suction $(s>0)$ and the effect of injection $(s<0)$ is just the opposite. Like the buoyancy parameter $\alpha$ or the magnetic parameter $M$, suction $(s>0)$ reduces both the momentum and thermal boundary layer thicknesses and the effect of injection $(s<0)$ is just the reverse. Hence $C_{\mathrm{f}}\left(\mathrm{Re}_{x}\right)^{1 / 2}$ and $\mathrm{Nu}\left(\mathrm{Re}_{x}\right)^{-1 / 2}$ increase with suction $(s>0)$.
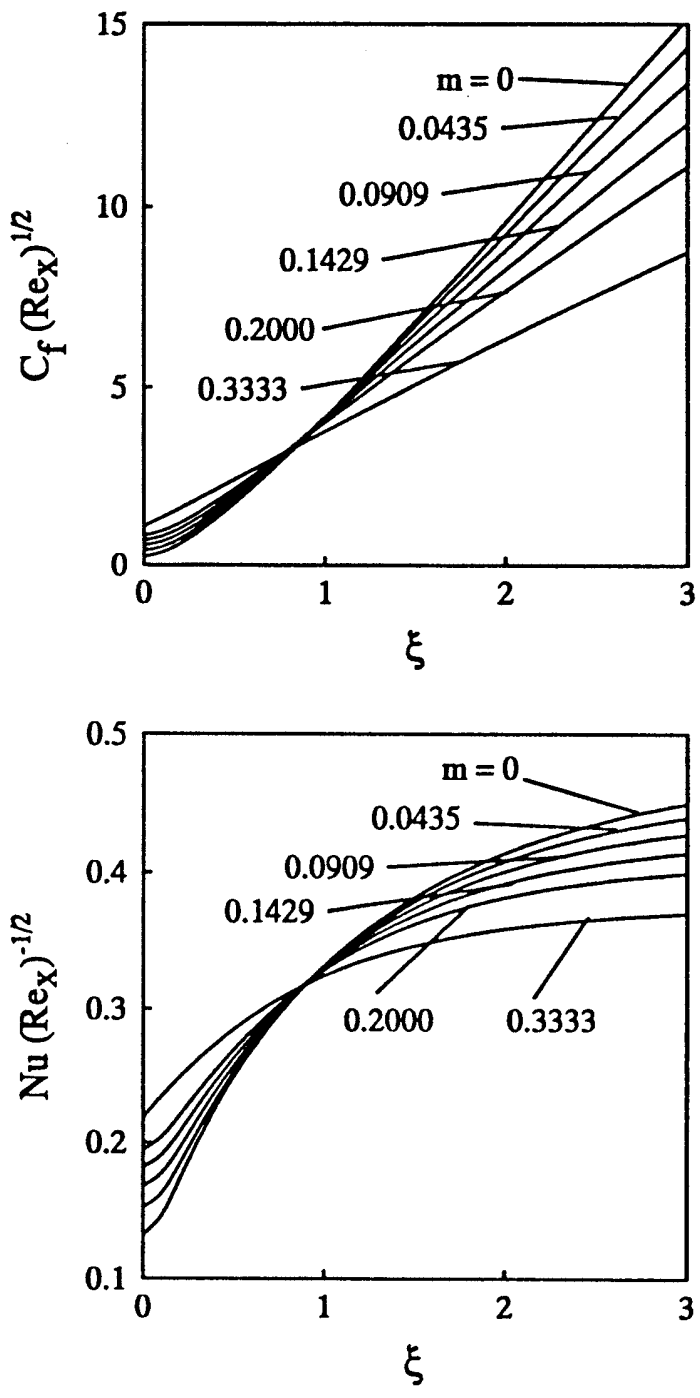

Fig. 9. Skin-friction coefficient $C_{\mathrm{f}}\left(\operatorname{Re}_{x}\right)^{1 / 2}$ and heat-transfer coefficient $\mathrm{Nu}\left(\mathrm{Re}_{x}\right)^{-1 / 2}$ for $K=0.5, s=-0.5, M=1, \alpha=2$ and $E=0$ and $\operatorname{Pr}=0.72$ 
The effect of the Prandtl number, Pr, on the skinfriction and heat-transfer coefficients $\left(C_{\mathrm{f}}\left(\operatorname{Re}_{x}\right)^{1 / 2}\right.$, $\left.\mathrm{Nu}\left(\mathrm{Re}_{x}\right)^{-1 / 2}\right)$ is shown in Fig. 8. It is seen that the heattransfer coefficient $\left(\mathrm{Nu}\left(\mathrm{Re}_{x}\right)^{-1 / 2}\right)$ increases with $\mathrm{Pr}$, but the skin-friction coefficient $C_{\mathrm{f}}\left(\mathrm{Re}_{x}\right)^{1 / 2}$ decreases. The reason for such a behaviour is that the higher Prandtl number fluid has a relatively low thermal conductivity which opposes conduction and thereby increases the variation. This results in the reduction of the thermal boundary layer thickness and an increase in the conductive heat transfer. On the other hand, the momentum boundary layer thickness increase with $\operatorname{Pr}$, because higher Prandtl number implies more viscous fluid. Consequently, the skin friction is reduced as $\mathrm{Pr}$ increases.

Figure 9 shows the effect of the pressure gradient parameter $(m)$ on the skin-friction coefficient $\left(C_{\mathrm{f}}\left(\mathrm{Re}_{x}\right)^{1 / 2}\right)$ and heat-transfer coefficient $\left(\mathrm{Nu}\left(\mathrm{Re}_{x}\right)^{-1 / 2}\right)$. It is observed that the effect of $m$ on $C_{\mathrm{f}}\left(\mathrm{Re}_{x}\right)^{1 / 2}$ and $\mathrm{Nu}\left(\mathrm{Re}_{x}\right)^{-1 / 2}$ becomes more pronounced as the distance $\xi$ increases. Also both $C_{\mathrm{f}}\left(\mathrm{Re}_{x}\right)^{1 / 2}$ and $\mathrm{Nu}\left(\mathrm{Re}_{x}\right)^{-1 / 2}$ increase as $\xi$ in-
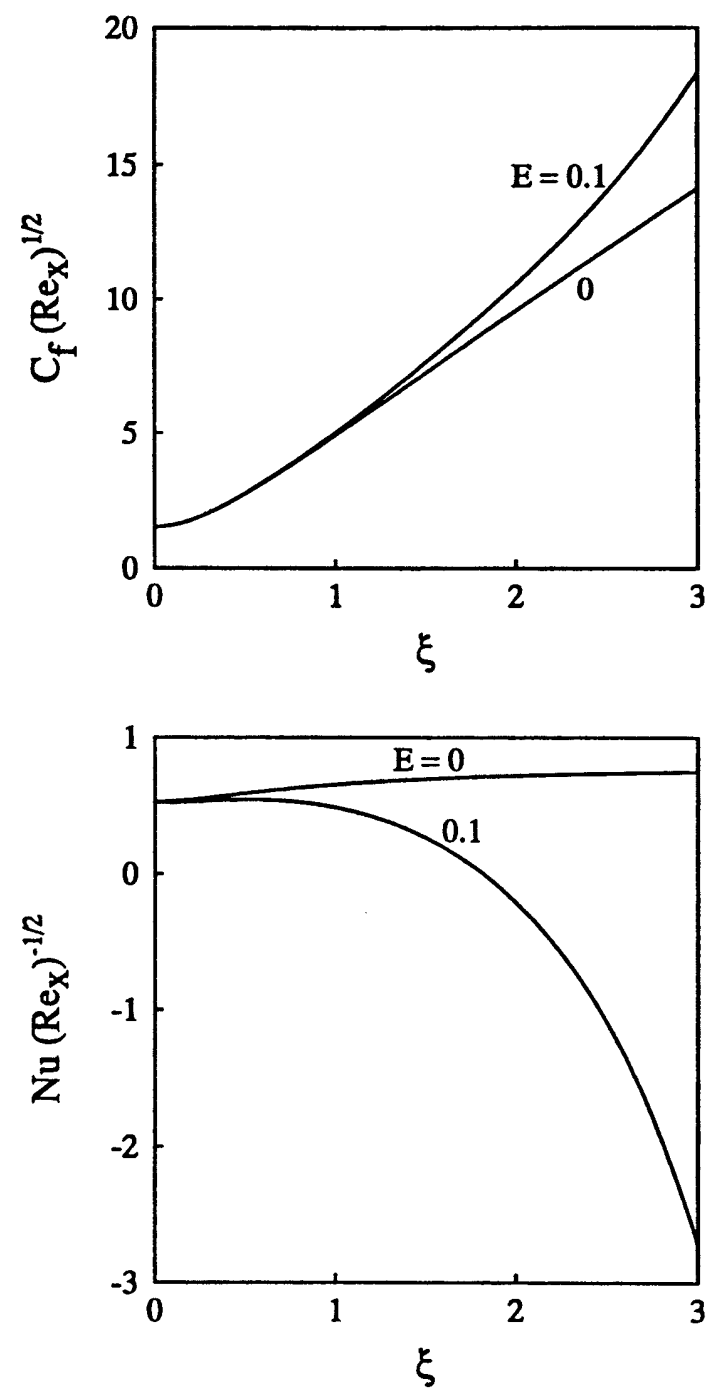

Fig. 10. Skin-friction coefficient $C_{\mathrm{f}}\left(\mathrm{Re}_{x}\right)^{1 / 2}$ and heat-transfer coefficient $\mathrm{Nu}\left(\mathrm{Re}_{x}\right)^{-1 / 2}$ for $K=s=0.5, m=0.0909, M=1$, $\alpha=2$ and $\operatorname{Pr}=0.72$ creases. It is also observed that $C_{\mathrm{f}}\left(\mathrm{Re}_{x}\right)^{1 / 2}$ and $\mathrm{Nu}\left(\mathrm{Re}_{x}\right)^{-1 / 2}$ increase as $m$ increases till certain $\xi$, say $\xi^{*}$. For $\xi>\xi^{*}$, these decrease with the pressure gradient parameter $m$.

The effect of the viscous dissipation parameter $E$ on the skin-friction and heat-transfer coefficients $\left(C_{\mathrm{f}}\left(\mathrm{Re}_{x}\right)^{1 / 2}\right.$, $\left.\mathrm{Nu}\left(\mathrm{Re}_{x}\right)^{-1 / 2}\right)$ is shown in Fig. 10. The effect of $E$ on $C_{\mathrm{f}}\left(\mathrm{Re}_{x}\right)^{1 / 2}$ and $\mathrm{Nu}\left(\mathrm{Re}_{x}\right)^{-1 / 2}$ becomes more pronounced as $\xi$ increases. It may be noted that for $\xi=0$, the terms containing $E$ in Eq. (12) vanishes. The effect of $E$ is more pronounced on the heat-transfer $\left(\mathrm{Nu}\left(\mathrm{Re}_{x}\right)^{-1 / 2}\right)$ than on the skin-friction $\left(C_{\mathrm{f}}\left(\mathrm{Re}_{x}\right)^{1 / 2}\right)$, because $E$ occurs explicitly in Eq. (12) which represents the equation for dimensionless temperature $G$.

The effects of the buoyancy parameter $\alpha$ and streamwise distance $\xi$ on the velocity and temperature profiles $\left(f^{\prime}, G\right)$ are shown in Fig. 11. We find that there is an overshoot in the velocity for the aiding flow $(\alpha>0)$ which increases as $\alpha$ or $\xi$ increases. There is no velocity overshoot either for the forced convection flow $(\alpha=0)$ or for the opposing flow $(\alpha<0)$. The reason for the overshoot in the velocity
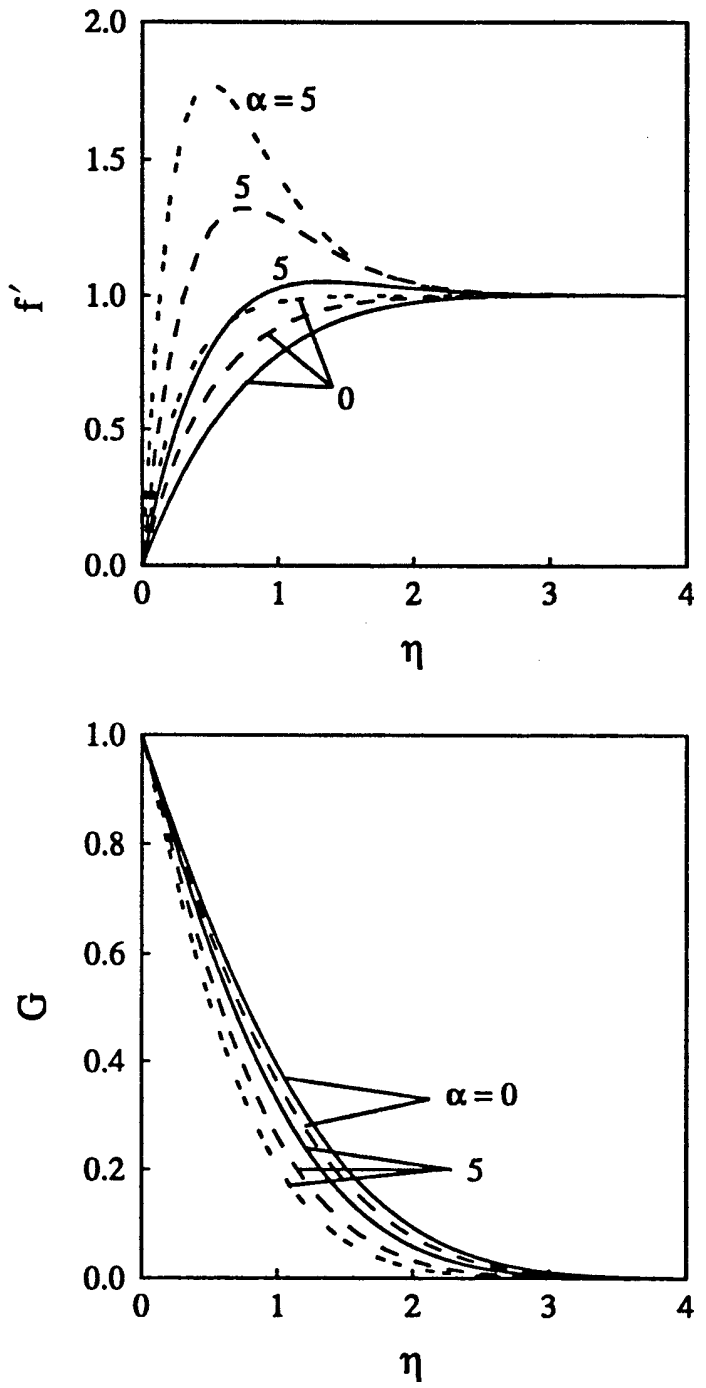

Fig. 11. Velocity profile $f^{\prime}$ and temperature profile $G$ for $K=s=0.5, m=0.0909, M=1, E=0$ and $\operatorname{Pr}=0.72$. $\xi=0.5 ;---: \xi=1 ;-\cdots--: \xi=2$ 
is that the buoyancy force $(\alpha>0)$ gives rise to a favourable pressure gradient resulting in velocity which adds to the forced convection velocity. The buoyancy opposed flow $(\alpha<0)$ gives rise to adverse pressure gradient which reduces the forced convection velocity. Hence no velocity overshoot is observed for the opposing flow $(\alpha<0)$. The temperature profiles $(G)$ show that for the case of aiding flow $(\alpha>0)$, an increase in the buoyancy parameter $\alpha$ results in an increase in the temperature gradient at the wall due to the reduction in the thermal boundary layer thickness. For the buoyancy opposed flow $(\alpha<0)$, the effect is just the reverse. It is also observed that the thermal boundary layer thickness decreases with the streamwise distance $\xi$.

\section{4}

\section{Conclusions}

The permeability, buoyancy, pressure gradient and magnetic parameters strongly affect the skin friction, but their effects on the heat transfer is comparatively less. The mass transfer strongly affects both the skin friction and the heat transfer. Also the heat transfer strongly depends on the dissipation parameter. The heat transfer is found to increase with the Prandtl number but the skin friction decreases. The buoyancy force which assists the forced convection flow causes an overshoot in the velocity profile.

\section{References}

Bejan A; Khair KR (1985) Heat and mass transfer by natural convection in a porous medium. Int J Heat Mass Transfer 28: $909-918$

Bejan A (1987) The basic scales of natural convection heat and mass transfer in fluids and fluid saturated porous media. Int Comm Heat Mass Transfer 14: 107-123

Cebeci T; Bradshaw P (1984) Physical and Computational Aspects of Convective Heat Transfer. Springer-Verlag, New York, pp. 406-415

Chen HT; Chen CK (1988) Free convection flow on nonNewtonian fluids along a vertical plate embedded in a porous medium. J Heat Transfer 110: 257-260

Cheng P (1977) Combined free and forced convection flow about inclined surfaces in porous media. Int J Heat Mass Transfer 20: $807-814$
Cheng P; Minkowycz WJ (1977) Free convection about a vertical flat plate embedded in a porous medium with application to heat transfer from a dyke. J Geophys Res 82: 2040-2044

Cheng P (1978) Heat transfer in geothermal system. Adv Heat Transfer 14: 1-105

Cheng P (1985) Natural convection in a porous medium: external flows. Proc NATO Adv Study on Natural Convection, Izmir, Turkey

Evans HL (1968) Laminar Boundary Layer Theory. Addison Wesley, London, pp. 168-171

Ingham DB; Pop I (1987) Free convection from a semi-infinite vertical surface bounded by a horizontal wall in a porous medium. Int J Heat Mass Transfer 30: 1615-1622

Merkin JH, Pop I (1987) Natural convection about twodimensional bodies with uniform surface heat flux in a porous medium. Acta Mechanica 70: 235-242

Ram PC (1988) Hall effects on the hydromagnetic free convective flow and mass transfer through a porous medium bounded by an infinite vertical porous plate with constant heat flux. Int J Energy Res 12: 229-231

Ramachandran N; Chen TS; Armaly BF (1988) Mixed convection in stagnation flows adjacent to vertical surfaces. J Heat Transfer 110: 373-377

Raptis A; Kafousias N (1982) Heat transfer in flow through a porous medium bounded by an infinite vertical plate under the action of a magnetic field. Int J Energy Res 6: 241-245

Raptis A (1986) Flow through a porous medium in the presence of a magnetic field. Int J Energy Res 10: 97-100

Raptis A; Perdikis CP (1988) Combined free and forced convection flow through a porous medium. Int J Energy Res 12: $557-560$

Takhar HS; Perdikis CP (1986) Forced and free convection flow of water at $4{ }^{\circ} \mathrm{C}$ through a porous medium. Int Comm Heat Mass Transfer 13: 605-609

Vafai K; Tien CL (1981) Boundary and inertia effects on flow and heat transfer in porous media. Int J Heat Mass Transfer 24: 195-203

Watanabe T (1990) Thermal boundary layers over a wedge with uniform suction or injection in forced flow. Acta Mechanica 83: 119-126

Watanabe T (1991) Forced and free mixed convection boundary layer flow with uniform suction or injection on a vertical flat plate. Acta Mechanica 89: 123-132 